\title{
Unsteady Aerodynamics of Single and Tandem Wheels
}

\author{
Stefano Spagnolo ${ }^{\mathrm{a}}$, Xin Zhang ${ }^{\mathrm{b}, *}$, Zhiwei Hu$^{\mathrm{a}}$, Oksana Stalnov ${ }^{\mathrm{c}}$, David \\ Angland ${ }^{\mathrm{a}}$ \\ ${ }^{a}$ Airbus Noise Technology Centre, University of Southampton, Southampton, SO16 7QF, \\ $U K$ \\ ${ }^{b}$ The Hong Kong University of Science and Technology, Clear Water Bay, Kowloon, Hong \\ Kong SAR, China \\ ${ }^{c}$ Faculty of Aerospace Engineering, Technion - Israel Institute of Technology, Haifa 32000, \\ Israel
}

\begin{abstract}
The major unsteady aerodynamic forces and major physics of a generic single wheel and tandem wheels are studied for the first time using wind tunnel tests. The wind-tunnel tests are performed in the $2.1 \mathrm{~m} \times 1.5 \mathrm{~m}$ wind tunnel at the University of Southampton. The tandem-wheel configuration consists of two in-line wheels that can be tested at different inter-axis distances and various installation angles. A vibration test is performed in situ on the model assembly to validate the unsteady-load measurements. Mean and unsteady aerodynamic loads and on-surface pressures are measured. Particle Image Velocimetry is used to acquire the velocity fields in the wake downstream of the model and surface oil-flow technique is used to identify the flow features on the surface of the wheels. Proper Orthogonal Decomposition is also used to characterise the wake in terms of unsteady fluctuations. The results of the experiments on the tandem wheels show that higher values of inter-axis distance correspond to slightly higher total mean drag coefficients and remarkably lower drag coefficient RMS values. Higher installation angles are associated with higher mean drag coefficients but generally lower fluctuations of the force coefficients. Non-zero mean lift coefficients are found for low inter-axis distance configurations at zero installation angle. The flow on the single wheel and on the front wheel of the tandem wheels is affected by laminar-turbulent transitional features. The vortical structures past the tandem wheels consist of four vortices that detach from the tyre shoulders of the front wheel and interact with the rear wheel. The study and obtained databases contribute to the general understanding of the complex flow and help to improve engineering predication of the gear aerodynamic loads.
\end{abstract}

Keywords: tandem wheels, aerodynamic forces, unsteady, aerodynamics, PIV, POD

\footnotetext{
${ }^{*}$ Corresponding author

Email address: aexzhang@ust.hk (Xin Zhang)
} 


\section{Nomenclature}

$C_{D} \quad$ Drag coefficient (x-axis), $C_{D}=F_{x} /\left(\rho S U_{\infty}^{2} / 2\right)$

$C_{L} \quad$ Lift coefficient ( $y$-axis), $C_{L}=F_{y} /\left(\rho S U_{\infty}^{2} / 2\right)$

$C_{p} \quad$ Pressure coefficient $C_{p}=\left(p-p_{\infty}\right) /\left(\rho U_{\infty}^{2} / 2\right)$

$D_{w} \quad$ Wheel diameter, $\mathrm{m}$

F Force vector, N

$k \quad$ Turbulent kinetic energy (2D): $k=\left(\overline{\left(u_{x}^{\prime}\right)^{2}}+\overline{\left(u_{y}^{\prime}\right)^{2}}\right) / 2, \mathrm{~m}^{2} / \mathrm{s}^{2}$

$L_{w} \quad$ Inter-axis distance, $\mathrm{m}$

$N \quad$ Number of PIV frames

$p \quad$ Pressure, $\mathrm{Pa}$

$R \quad$ Wheel-shoulder radius, $\mathrm{m}$

$R e_{D} \quad$ Reynolds number

$S \quad$ Frontal projected wheel area $S=0.01285 \mathrm{~m}^{2}$

St Strouhal number

T Transfer function

U Velocity, $\mathrm{m} / \mathrm{s}$

$x, y, z$ Global coordinate system, $\mathrm{m}$

$z_{c} \quad$ Confidence coefficient

$\infty \quad$ Free-stream value

$p \quad$ PIV axis

RMS Root Mean Square

$\alpha \quad$ Installation angle, deg

$\epsilon_{\mu} \quad$ Relative error on the mean

$\epsilon_{\sigma} \quad$ Relative error on the standard deviation

$\nu \quad$ Kinematic viscosity of air, $\mathrm{m}^{2} / \mathrm{s}$

$\rho \quad$ Density of air, $\mathrm{kg} / \mathrm{m}^{3}$

$\theta \quad$ Wheel azimuthal angle, deg

Fluctuating part 
- Time average

$f \quad$ Front wheel

$r \quad$ Rear wheel

$t \quad$ Total

\section{Introduction}

Most of the existing research on the flow past landing gears focuses on aeroacoustics because of the increasingly stringent noise requirements and due to the fact that landing gears are still one of the main sources of noise of civil aircraft [6] during the approach and landing phases. Obtaining a good understanding of the unsteady flow features is not only useful in order to predict noise, but also to correctly estimate the mean and unsteady aerodynamic forces.

For a rudimentary four-wheel landing gear, mean lift, drag and side forces were measured by Venkatakrishnan et al. [32. It was found that the strut strongly affects the flow field around the two in-line wheels. Lazos [12] measured the flow velocity in a streamwise plane surrounding the wheels of a four-wheel undercarriage by particle image velocimetry (PIV). The measurement identified different flow states at low frequency. Stalnov et al. 28] experimentally studied a simplified quarter-scale model of a main landing gear. In addition to aeroacoustic measurements, mean aerodynamic loads were measured sequentially on sub-assemblies of the landing gear, from an isolated main strut to a full assembly, mounting the components sequentially. In this way it was possible to analyse the effect of the various components on the landing-gear aerodynamic loads and noise, but it was not possible to isolate the effects of the interactions between wheels and struts. The model was studied for multiple installation angles of the bogie. Other works on aircraft undercarriages were performed on the Boeing 777 main landing gear (Humphreys and Brooks 9]), on the Gulfstream G550 nose landing gear (Zawodny et al. 34]), and on the Airbus LAGOON nose landing gear (Manoha et al. [15]). However, unsteady aerodynamic loads were not quantified experimentally in any of these studies.

In contrast to the studies described above, in which complex assemblies are considered, there are a number of studies on elementary geometries, representing parts of a landing gear. These studies are generally meant to provide a better understanding of the flow features. For instance the work by Khorrami et al. [10] provided experimental data on tandem cylinders 29, 19. On the same tandemcylinders geometry, Xiao and Luo 33 tested the Improved Delayed DetachedEddy simulations (IDDES) by Shur et al. 24]. A dedicated tandem-cylinder benchmark case was extensively tested in the NASA low-speed aeroacoustic wind tunnel to provide a database for aeroacoustic predictions (Lockard [13]), followed by simulations on the same geometry.

The flow past isolated wheels is similar to the flow past small-aspect-ratio cylinders. Zdravkovich et al. 35. experimentally studied the flow past smallaspect-ratio cylinders with two free ends in the critical regime $2 \times 10^{5}<R e<$ 
$6 \times 10^{5}$. It was found that the wake is dominated by a vortex system that is formed by four vortex filaments. Similar flow features were noticed on more realistic wheel shapes too. McManus and Zhang [16] performed simulations on the flow past an isolated wheel in contact with both stationary and moving ground at a Reynolds number of $5.3 \times 10^{6}$. When the wheel is stationary on the ground, two opposite vortices form on the top wheel shoulders. On the contrary, when the wheel is rotating, the separated flow on the top part of the wheel forms an arch vortex layer. Later, Zhang et al. 36] experimentally studied the flow past the $1 / 3$-scale CADWIE isolated wheel with two different hubs that did not have any significant effect on the flow.

For the purpose of measuring both mean and unsteady aerodynamic loads, strain-gauge balances are commonly used 31. The strain is measured on the sensor structure and, after calibration, the forces are estimated. For example, unsteady data for wind aerodynamic loads on wind-turbine models were obtained with a strain-gauge based balance by $\mathrm{Hu}$ et al. [8, where the balance was able to capture the unsteady loads at the blade rotation speed and at the higher harmonic frequencies up to three times the rotation speed. In the literature, attempts with direct application of strain gauges to some relevant points of the models exist. For example, Schuster and Byrd [23] applied strain gauges but only the data from the pressure sensors were considered reliable due to the influence of the structural dynamic modes on the strain gauges. For short-duration force measurements (e.g., Robinson and Hannemann [21]), models and special calibration rigs have to be prepared. This is more complex than the previous case because the model is designed for the purpose of the dynamic calibration.

Another approach is to obtain the loads by integrating the measured surface pressure using pressure sensitive paint [5] and piezoelectric sensors for high frequency local information for both surface shear stress and surface pressure [18, 1, 4. In some specific cases, more complex techniques can be applied. For example, on two-dimensional geometries, time-resolved PIV can be used to estimate the forces on the model [11, whilst small-size models can be supported by a magnetic suspension system [22] that measures the forces.

Concerning the analysis of the flow features, Proper Orthogonal Decomposition (POD) has been used to extract the modes of the flow field, both in experiments and simulations. Examples are Meyer et al. [17, comparing PIV data with Large Eddy Simulations (LES), Bernero and Fiedler 3 on PIV data, and Tirunagari et al. 30] on LES data. There is the need of using such a technique because the turbulent flow in the wake is chaotic and random, and POD allows the identification of the choerent structures and their classification by energy content 2 .

Surface oil-flow is used as well to help identifying the mean flow structures on the surface. A description of the surface patterns is detailed in $\mathrm{Lu}$ [14]. Various examples of application of this technique are present in the literature: small aspect ratio cylinders [35, single wheel [36] and full landing gears [28. The surface flow visualization is used to confirm trends observed in the measurements and to have a global perspective of the flow on the analysed bodies, also in case of complex geometries. 
The current work aims to achieve a better understanding of mean and unsteady characteristics of the flow around landing-gear components. Loads, surface pressures, and velocity fields are measured on a new geometry that represents a simplified model of a landing gear component: wheels in single and tandem configurations. The tandem configuration represents half of a four-wheel bogie without any interference from struts, doors, bogie beams, axles, etc. A dedicated vibration test [20] is performed to assess the capability of the balance to measure unsteady loads. The vibration test allowed the assessment of the unsteady forces for the first time in the wind tunnel. To the authors' knowledge, it is the first time that the unsteady tests performed in the experiments were conducted. The studies are a part of a large study including tests, analysis and numerical simulations 27, 26. The overall objective of the study is to move the prediction of aerodynamic forces to a more mature stage in terms of accuracy and efficiency.

\section{Experimental Setup and Procedures}

\subsection{Test facility and model geometry}

The experiments were conducted at the University of Southampton, in a closed-circuit closed-test-section wind tunnel with a cross-section of $2.1 \mathrm{~m} \times$ $1.5 \mathrm{~m}$. The wind tunnel can be operated at free-stream velocities $U_{\infty}$ between $10 \mathrm{~m} / \mathrm{s}$ and $48 \mathrm{~m} / \mathrm{s}$, corresponding to a Reynolds number $R e_{D}=U_{\infty} D_{w} / \nu$ range between $1.2 \times 10^{5}$ and $5.8 \times 10^{5}$ for the current model $\left(D_{w}\right.$ is the wheel diameter and $\nu$ is the kinematic viscosity of air).

The current model consists of two wheels in tandem. The wheel is a generic model of a landing-gear wheel, as shown in Figure 1(a) The geometric parameters are given as follows: the wheel diameter $D_{w}$ is $0.181 \mathrm{~m}$, the width $W$ is $0.4 D_{w}$, and the fillet radius $R$ is $0.1 D_{w}$ (uniform). Measurements are performed in different configurations for three inter-axis distances $L_{w}\left(1.1 D_{w}, 1.3 D_{w}\right.$ and $\left.1.5 D_{w}\right)$ and three installation angles $\alpha(0 \mathrm{deg}, 10 \mathrm{deg}$ and $20 \mathrm{deg})$. The wheels are supported by streamlined struts to minimize the interference. A picture of the wheel model (mounted in the wind tunnel) is shown in Figure 1(b). The two supports are tapered zero-sweep half wings with a NACA-0024 cross section. The length of the support is $300 \mathrm{~mm}$ and its taper ratio (tip/root chord ratio) is 0.46 . The supports are mounted to have, for all the tested configurations, zero installation angle. The blockage ratio is between $0.5 \%$ and $1 \%$, depending on the specific configuration. The blockage is not expected to affect the flow and the measured data.

The model is manufactured by means of rapid prototyping techniques. In detail, the wheels are made by 3D printing with a LS600 photopolymer machine, while the supports are made of carbon-fibre reinforced plastic (CFRP) and aluminium alloy. The interfaces with the wind-tunnel frame are standard aluminium profiles plus a machined aluminium plate. The manufacturing tolerances for the external wheel surface can be estimated to be $\pm 0.1 \mathrm{~mm}$ with respect to the axis system of the $3 \mathrm{D}$-printing machine. The maximum error on the wheels position is $\pm 1 \mathrm{~mm}$. 
In this paper we focus the discussion to the untripped configurations with a flat hub cover (Figure 1(b) , to which all the data are referred unless otherwise specified. Details of the tests with various tripping devices and wheel covers other than the flat cover are given in Spagnolo et al. 27.

\subsection{Loads and pressure acquisition systems}

In the current work, the forces acting on the two wheels are measured separately. Aerodynamic forces are described in terms of both mean and unsteady values (i.e., RMS of the force signal).

A wind-tunnel balance would measure the combined force acting on the wheels and the supports. In order to isolate the force acting on the wheel, an internal force sensor (which measures only the loads acting on the wheels) is fitted inside one of the wheels. The force sensor is an ATI mini40, a 6-axis strain-gauge based transducer. The sampling time is approximately 60 seconds at a frequency of $1000 \mathrm{~Hz}$. The repeatability of the mean force coefficients is within 0.001 at a speed of $40 \mathrm{~m} / \mathrm{s}$.

An additional wheel model with identical external shape was manufactured for on-surface pressure data collection. This additional model was designed to host a miniature pressure scanner with multiple channels (instead of the balance) inside the wheel. The pressure scanner is the miniature 64-channels Scanivalve ZOC33. The model is manufactured with 21 equally-spaced holes for pressure taps along a 120-deg sector along the center-line circumference. Rotating the wheel four times (90 deg rotation each) allows for acquiring the full circumferential pressure data with a $30 \mathrm{deg}$ overlap for each rotating position. Overall, surface pressure data are available for 60 points equally spaced in the azimuthal angle $\theta$ on the full center-line circumference, with $\theta=0 \mathrm{deg}$ representing the most windward point on the circumference. The static reference pressure $p_{\infty}$ and the reference dynamic pressure $\rho U_{\infty}^{2} / 2$ are directly provided by a Pitot tube positioned in the test section. Two different sampling frequencies were tested, $113.6 \mathrm{~Hz}$ and $568.2 \mathrm{~Hz}$ for each channel, and no difference was found in the results.

In total, there are three wheel models available: one wheel is equipped with the pressure sensors, one wheel is equipped with force sensors and the third is not instrumented. Pressure measurements and force measurements are not performed simultaneously because it was not possible to embed the balance and the pressure sensor unit at the same time inside of the wheel. Normally during data acquisition, when testing the wheels in a tandem configuration, only one wheel is instrumented while the other wheel is not, then the two wheels are swapped to acquire the data for the other wheel position.

\subsection{Vibration tests and unsteady loads}

The strain-gauge based balance inside the wheel model quantifies the forces by means of the strain of the balance itself. For dynamic load measurements, at high frequencies the measurments are affected by the dynamic response of the model assembly [20. In addition, the dynamic response is generally different in 
each direction, and the modes are coupled: this means that even when the model is forced in one direction, the balance produces non-zero dynamic values in the other components. Here, the aerodynamic unsteady loads are validated by a vibration test to identify the model dynamic response. The vibration test must be performed in situ: the model is mounted in the wind tunnel and an electromagnetic shaker is used to force the model at different spots. The vibrationtest equipment consists of a Data Physics Signal-Force GW-V4/PA30E shaker and a Dytran 1053V piezoelectric force sensor. The shaker can provide both a random and a sinusoidal force, which is transmitted to the model through the piezoelectric force sensor.

The force measured by the force sensor inside the model and the excitation force are simultaneously acquired. To understand the accuracy of the measured dynamic values, the transfer function $T$ between the external forces and the measured values from the balance is calculated. The transfer functions for excitation forces along the two axes $x$ and $y$ are given in Figure 2, which shows that the unsteady load data measured by the balance are consistent with the externally applied loads up to approximately $30 \mathrm{~Hz}$. The transfer functions were obtained in the wind-tunnel for one configuration only, but similar transfer functions can be assumed for the other configurations since the only difference is the position of the supports on the mounting plate, which has minimal effects on the dynamic response of the structure.

The transverse sensitivity can be associated with modes coupling phenomena. Positioning or alignment errors in the shaking system, and additional modes relative to the wind tunnel mountings or to minor features of the model may also play a small part. The transverse sensitivity (not shown) is within 15\% of the external load. The transfer functions of the moments show analogous results, with less accuracy, due to the lower intensity of the moments applied. The current work considers only the $F_{x}$ and $F_{y}$ forces, both in terms of mean loads and unsteady loads, therefore the transfer functions of the moments are not investigated any further.

The vibration test allows the calculation of the cut-off frequency on the basis of the model response with a clear identification of the ratios between the external forces and the measured forces, for each axis.

\subsection{Particle Image Velocimetry}

The PIV setup uses a single 4 Megapixel camera equipped with a Sigma lens of focal length $105 \mathrm{~mm}$ to acquire the two in-plane components within a square frame behind the rear wheel. The reference axes $x_{p}-y_{p}$ for the PIV frames are positioned at the most downstream point of the rear wheel, as illustrated in Figure 1(a) For single-wheel configurations the front wheel is removed. The flow fields are measured only at a free-stream velocity $U_{\infty}=40 \mathrm{~m} / \mathrm{s}$ (corresponding to $\left.R e_{D}=4.8 \times 10^{5}\right)$ on the wheel center-line plane $(z=0)$, in Figure $1(\mathrm{a})$

The camera is mounted at a distance of approximately $1.8 \mathrm{~m}$ from the target plane, outside the wind tunnel. The laser, a Quantel Twins BSL 200, is configured to fire two consecutive shots with a $20 \mu$ s delay. The frame acquisition 
is repeated at a frequency of $3.63 \mathrm{~Hz}$ for 500 frames. The results reported in Section 3 are shown for a frame of $200 \mathrm{~mm} \times 200 \mathrm{~mm}$.

To estimate the maximum relative errors, $\epsilon_{\mu}$ and $\epsilon_{\sigma}$, on the resulting mean velocity component $\bar{U}_{i}$ and its standard deviation $U_{\mathrm{RMS}, i}$, the following formulas (Grant and Owens [7]) are applied:

$$
\begin{gathered}
\epsilon_{\mu}=\frac{z_{c} U_{\mathrm{RMS}, i}}{\bar{U}_{i} \sqrt{N}} \\
\epsilon_{\sigma}=\frac{z_{c}}{\sqrt{2 N}}
\end{gathered}
$$

where $N$ is the number of samples and $z_{c}$ is the confidence coefficient (here assumed equal to 1.96 for a $95 \%$ confidence level).

For the current tests, the error on the mean fields is lower than $1.6 \mathrm{~m} / \mathrm{s}$ and the error on the standard deviation is within $1 \mathrm{~m} / \mathrm{s}$ (both with a confidence level of $95 \%$ ). The errors are higher in the region close to the wheel, and become smaller further away. This is because the turbulence level is high, affecting the statistical estimation of the error in Equations 1 and 2

\subsection{Proper Orthogonal Decomposition}

The POD is applied to the velocity fields from PIV to recognize the most energetic patterns in the wake. The POD methodology used is the snapshot POD method by Sirovich [25]. The analysis is performed on the fluctuating part of the two velocity $\left(u_{j}^{n}, v_{j}^{n}\right)$ components captured in the PIV, where $j$ is the index of the point and $n$ is the index of the frame number. The velocity components are ordered in a matrix $\mathbf{U}$ of size $2 M \times N$, where $M$ is the number of velocity components for each snapshot and $N$ is the number of acquired snapshots.

$$
\mathbf{U}=\left[\mathbf{u}^{1} \mathbf{u}^{2} \ldots \mathbf{u}^{N}\right]=\left[\begin{array}{cccc}
u_{1}^{1} & u_{1}^{2} & \ldots & u_{1}^{N} \\
\vdots & \vdots & \vdots & \vdots \\
u_{M}^{1} & u_{M}^{2} & \ldots & u_{M}^{N} \\
v_{1}^{1} & v_{1}^{2} & \ldots & v_{1}^{N} \\
\vdots & \vdots & \vdots & \vdots \\
v_{M}^{1} & v_{M}^{2} & \ldots & v_{M}^{N}
\end{array}\right]
$$

The eigenvalue problem

$$
\tilde{\mathbf{C}} \mathbf{A}^{i}=\lambda^{i} \mathbf{A}^{i}
$$

has to be solved. $\tilde{\mathbf{C}}$ is the autocovariance matrix that is defined as $\tilde{\mathbf{C}}=\mathbf{U}^{t} \mathbf{U}$. After reordering the eigenvalues in decreasing order, the eigenvectors can be used to generate the POD modes:

$$
\phi^{i}=\frac{\sum_{n=1}^{N} A_{n}^{i} \mathbf{u}^{n}}{\left\|\sum_{n=1}^{N} A_{n}^{i} \mathbf{u}^{n}\right\|}
$$


with $i=1, \ldots, N$, and $A_{n}^{i}$ the $n$-th component of the eigenvector corresponding to $\lambda_{i}$. The discrete 2 -norm is defined as:

$$
\|y\|=\sqrt{y_{1}^{2}+y_{2}^{2}+\cdots+y_{M}^{2}}
$$

The POD modes $\phi^{i}$ can be grouped in a matrix $\mathbf{\Phi}=\left[\phi^{1} \phi^{2} \cdots \phi^{N}\right]$. The POD coefficient $a_{i}$ for the $i$-th mode can be obtained by projecting the fluctuating velocity components onto the POD modes:

$$
\mathbf{a}^{n}=\boldsymbol{\Phi}^{t} \mathbf{u}^{n}
$$

Outcome of the POD analysis is presented in 3.3 These include the energy contents associated with the modes and the cumulative energy content for both the single wheel and the tandem wheels.

\subsection{Surface oil-flow visualization}

In performing oil-flow visualization, a kerosene-based visualization liquid is applied on the surface of the wheels and the wind tunnel is started and operated for approximately $10-15$ minutes at $R e_{D}=4.8 \times 10^{5}$. The pigments left on the surfaces after each run allows the identification of the surface streamlines. 


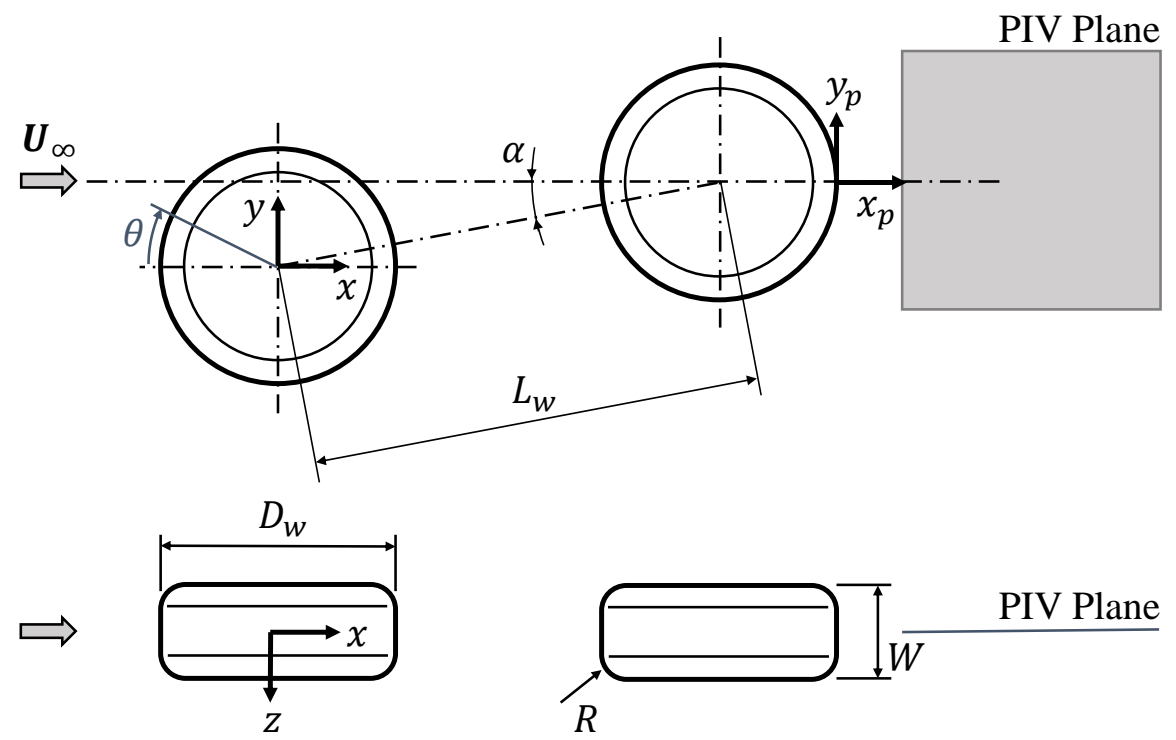

(a) Schematic drawing of a generic tandem-wheel configuration.

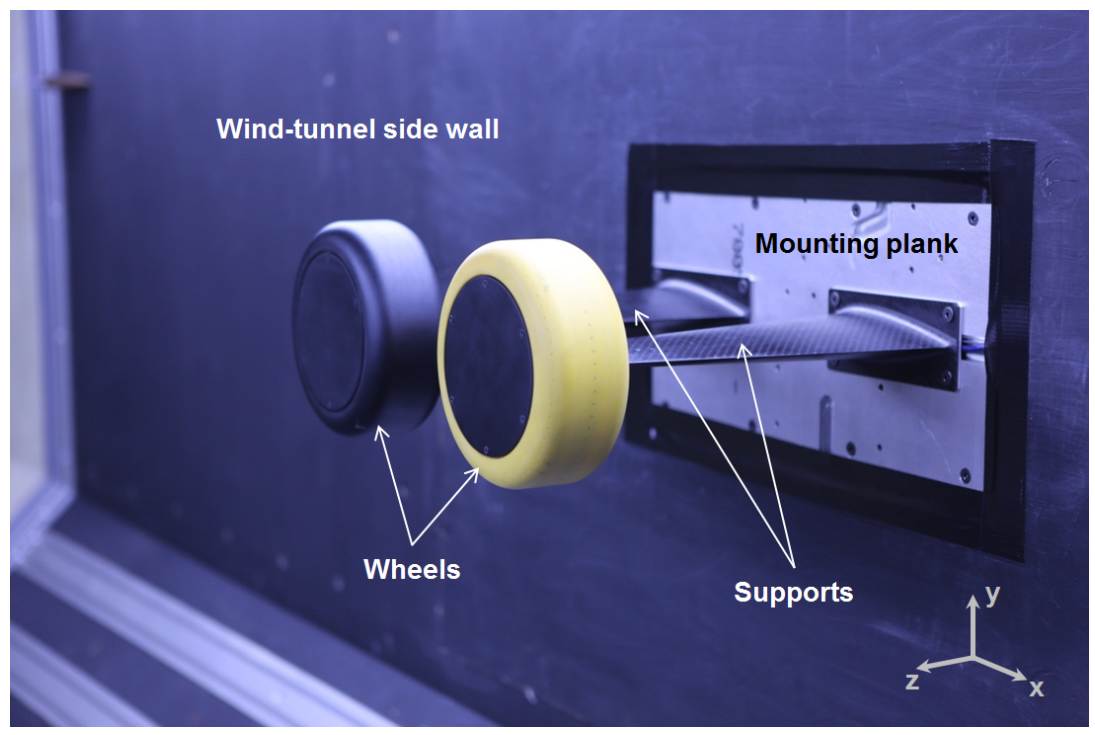

(b) Picture of the wind-tunnel model assembly.

Figure 1: Tandem-wheels experimental assembly with the main geometric parameters and the global reference system ( $x$ the streamwise, $y$ the vertical, and $z$ the spanwise direction). 


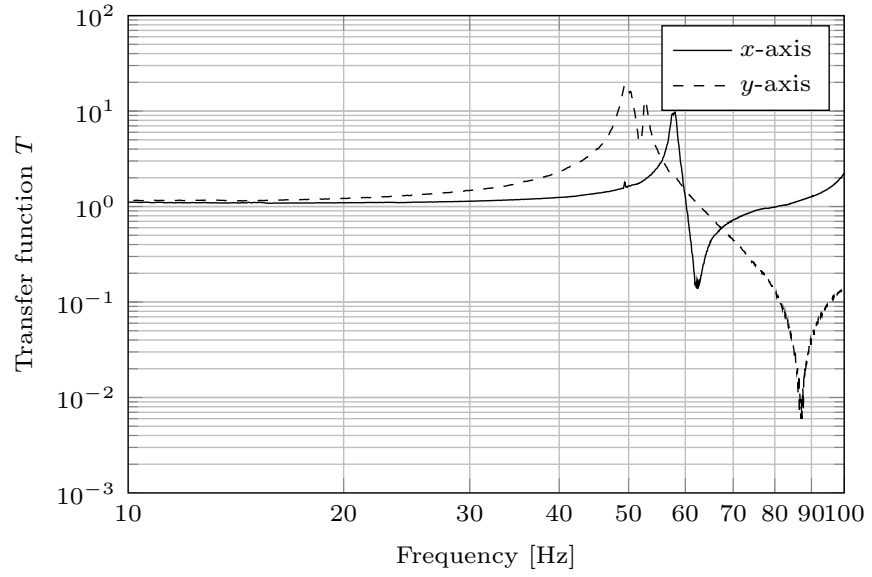

Figure 2: Transfer function $T$ from the vibration test with external force applied along the $x$-axis and along the $y$-axis. 


\section{Results and Discussion}

\subsection{Effects of Reynolds number}

The model was tested up to a Reynolds number $\operatorname{Re}_{D}$ of $5.7 \times 10^{5}$, which is approximately one order of magnitude lower than a full-scale landing-gear. In this section, the effect of Reynolds number on the flow past a single wheel and tandem wheels is analysed. The mean drag coefficients $\bar{C}_{D}$ of the single wheel and one reference configuration of tandem wheels $\left(L_{w}=1.5 D_{w}\right.$ and $\alpha=0 \mathrm{deg})$ are shown as functions of Reynolds number $\left(R e_{D}\right)$ in Figure 3 For both the single wheel and the tandem wheels, the mean drag coefficient decreases with Reynolds number. For Reynolds numbers in the range $3.6 \times 10^{5}$ $<R e_{D}<5.7 \times 10^{5}$, the mean drag coefficient is approximately constant for the rear wheel in the tandem configuration, although the drag does continue to decrease slightly with an increase in Reynolds number for the single wheel and the front wheel in the tandem wheel configuration. The RMS of the force coefficients show similar dependency on Reynolds number (not shown here).

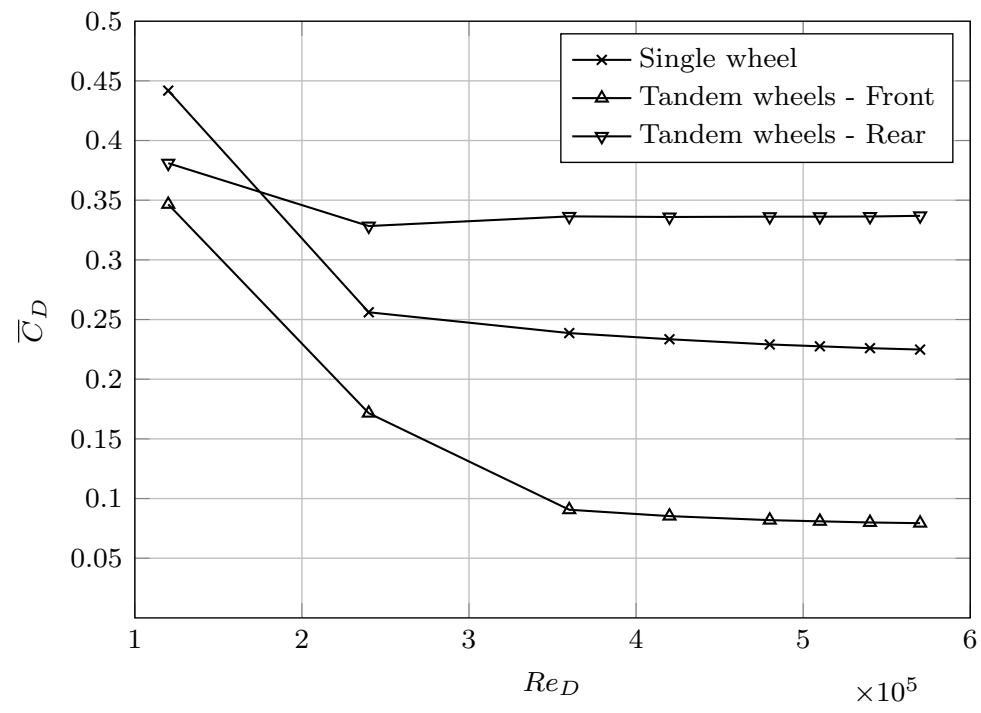

Figure 3: Mean drag coefficient $\bar{C}_{D}$ as a function of Reynolds number $R e_{D}$ for single and tandem wheels $\left(L_{w}=1.5 D_{w}\right.$ and $\left.\alpha=0 \mathrm{deg}\right)$, untripped.

The flow is dependent on Reynolds number because of the laminar flow features, appearing on the wheel surface in this flow regime. The evolution of the pressure coefficients on the wheel centreline with increasing Reynolds numbers is given in Figure 4 for the single-wheel case. Similarly to what happens on a circular cylinder, the position of the suction peaks moves towards the rear of the wheel with more pronounced peaks for increasing Reynolds numbers until the value stabilizes at $\bar{C}_{p}=-0.9$ above $R e_{D}=3.6 \times 10^{5}$, which is the same Reynolds number that marks the beginning of the plateau of the curve in 
Figure 3. The two blips on the curves of $\bar{C}_{p}$ (located at approximately $120 \mathrm{deg}$ and $240 \mathrm{deg}$ ) and the corresponding peaks of $C_{p, \mathrm{RMS}}$ indicate the presence of two recirculation bubbles, also identifiable by the plateau in the pressure coefficient, which is a typical feature of separation bubbles. In fact, a recirculation bubble is found on the wheel top and bottom surfaces, as it can be observed also from the surface oil-flow visualization in Figure 6. The top bubble is located at approximately $100 \mathrm{deg}<\theta<120 \mathrm{deg}$ on the wheel surface. The bubble indicates laminar-turbulent transition, where with increasing Reynolds number the size of the bubble reduces.

In order to understand to what extent this feature affects the flow, pressure coefficients $C_{p}$ for the untripped and the tripped configurations of the single wheel are compared in Figure 5 at $\operatorname{Re}_{D} \approx 4.8 \times 10^{5}$ (more details can be found in Spagnolo et al. 27]). The tripping technique used is a zig-zag tape on the front part of the wheel. The separation bubbles are present in the untripped configuration only, at approximately $120 \mathrm{deg}$ and $240 \mathrm{deg}$, as it has been previously shown. Instead, in the tripped configuration, these features are not seen in the $\bar{C}_{p}$ plot, whilst it is still possible to observe the corresponding peaks in the $C_{p, \text { RMS }}$ plots. In Figure 6, the recirculation bubble on the untripped configuration is shown with the oil-flow visualization. The change of the surface flow features between the untripped and the tripped configurations can be seen also in Figure 7, where the picture at the top for the untripped single wheel reveals the presence of the separation bubble at $100 \mathrm{deg}<\theta<120 \mathrm{deg}$ followed by the reattachment of the boundary layer. The flow does not show the same feature for the tripped case (bottom picture). Instead a similar recirculation area is present but it is in the separation region for $\theta>130 \mathrm{deg}$. The added tripping promotes the flow transition and keeps it attached for longer.

Additional separation bubbles are located in the proximity of the wheel rim, as indicated in Figure 8. With the experimental apparatus for pressure measurements, the lateral separation bubble cannot be captured in the pressure coefficient profiles. It can only be seen in the oil-flow visualizations. Thus, the laminar separation bubbles are expected to be the main cause of the Reynolds dependency. On the tandem wheels, only the top separation bubble is visible on the rear wheel at high installation angles, as can be seen in Figure 9. This is because the top part of the wheel is not contained in the wake of the front wheel. As it was found in Figure 3, at low angles of attack, the variation of the force coefficients on Reynolds number is low because the flow impinging on the rear wheel is turbulent and no Reynolds-dependent feature is present. On the contrary, the separation bubbles are always present on the front wheel, which shows a curve similar to that of the single wheel. Also in this case, the bubbles can be observed on the plots of the pressure coefficients, in Figure 17(a) and in Figure 17(b), which is discussed in more details later in 3.4. The laminar separation bubbles are not visible in the configurations with tripping devices (not shown), similarly to what happens in the single wheel case.

As mentioned in Section 2, the single wheel was tested also with two additional versions of wheel hub cover. The mean drag coefficient for the single wheel at $40 \mathrm{~m} / \mathrm{s}$ was 0.238 with the cavity-only cover, while it was 0.241 with 

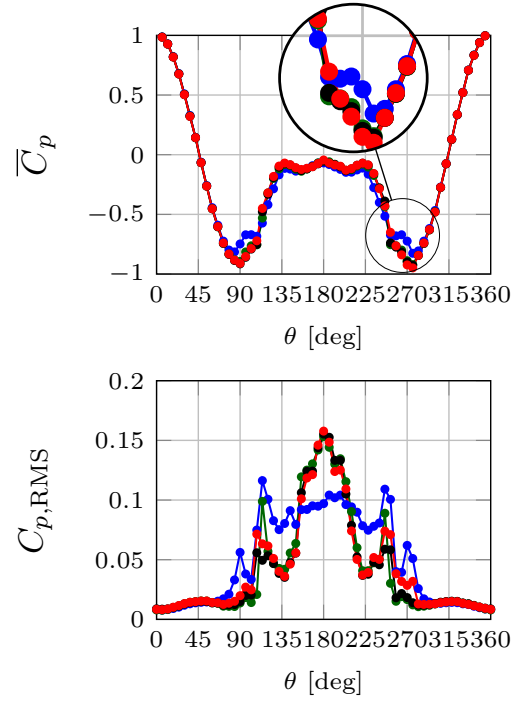

Figure 4: Single wheel, untripped case: mean and RMS values of $C_{p}$ along the wheel centre line, comparing various Reynolds numbers $R e_{D}$ : $2.7 \times 10^{5}$ $\because-3.6 \times 10^{5} \longrightarrow-\quad 4.8 \times 10^{5}$ $\longrightarrow$, and $5.6 \times 10^{5} \longrightarrow$.
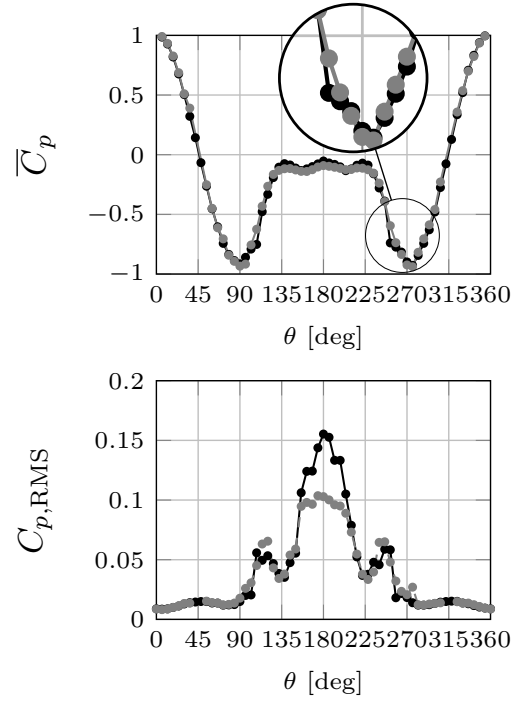

Figure 5: Single wheel $\left(R e_{D} \approx\right.$ $\left.4.8 \times 10^{5}\right)$ : mean and RMS values of the pressure coefficient $C_{p}$ along the wheel centre line, comparing the untripped $\rightarrow$ and the tripped $\rightarrow-\rightarrow$ case.

the cover including the simplified hub. Generally, the effect of the wheel hub covers is small, since it only introduces an increase in drag coefficient within 5\% from the baseline geometry with a flat cover. This is also confirmed by previous tests on the CADWIE wheel geometry [36. For this reason, no further tests were performed with this configuration.

Overall, the analysis of the flow features reveals that the flow on the single wheel and on the front wheel of the tandem wheels is affected by laminarturbulent transitional features, but the dependency on Reynolds number in the range $3.6 \times 10^{5}<R e_{D}<5.7 \times 10^{5}$ is generally small for the force coefficients. The rear wheel at low installation angles does not show Reynolds dependency for $R e_{D}>3.6 \times 10^{5}$. The data shown in the following sections of this paper are given for the untripped configurations. 


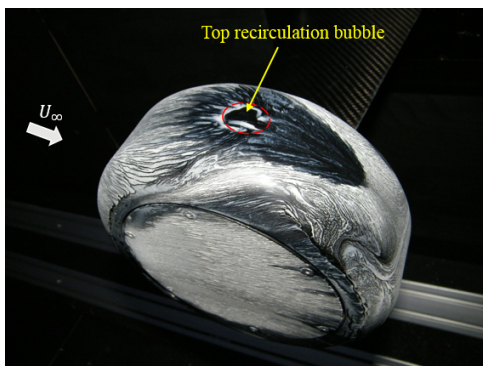

Figure 6: Oil flow on single wheel, top recirculation bubble, untripped configuration at $R e_{D}=4.8 \times 10^{5}$. The recirculation bubble on the bottom surface is similar.
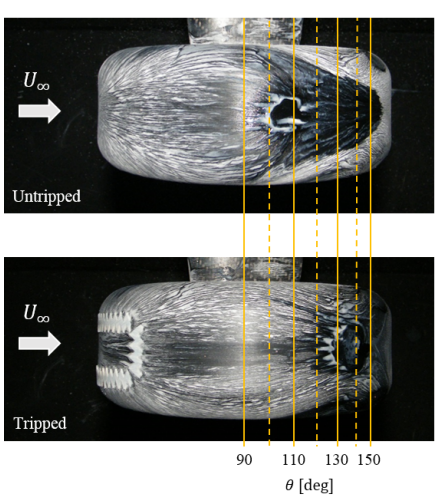

Figure 7: Oil flow on single wheel for both untripped and tripped configurations at $R e_{D}=4.8 \times 10^{5}$.

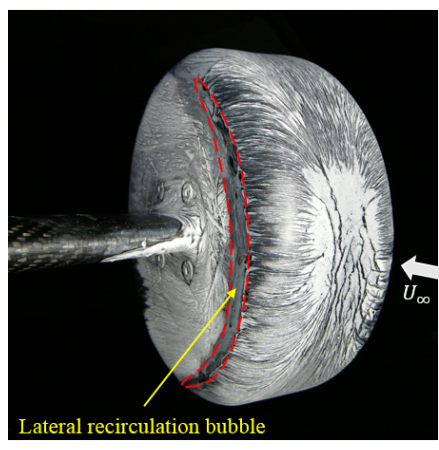

Figure 8: Oil flow on single wheel, lateral separation bubble, untripped configuration at $\operatorname{Re}_{D}=4.8 \times 10^{5}$.

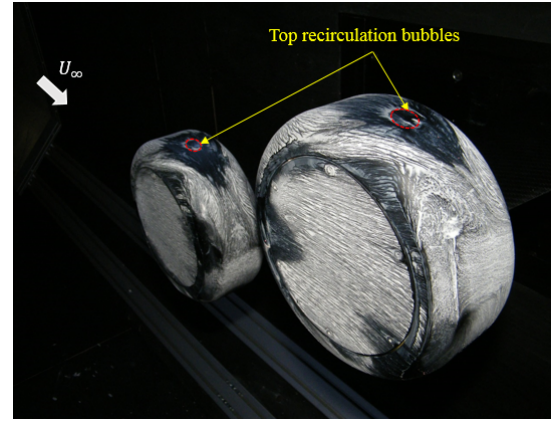

Figure 9: Oil flow on tandem wheels, $L_{w} / D_{w}=1.5$ and $\alpha=20 \mathrm{deg}$, at $\operatorname{Re}_{D}=$ $4.8 \times 10^{5}$. 


\subsection{Flow topology}

This section presents a detailed description of the wake structures. As it can be noted from Figure 10, the mean flow past the single wheel is made of four distinct vortices, which is similar to the flow observed by Zdravkovich et al. [35] on short-aspect-ratio cylinders. The adjacent vortices have opposite streamwise vorticity. Similarly, the flow past tandem wheels is made of four vortices. In Figure 10(a) the surface oil-flow pattern left by the vortices behind the single wheel can be clearly seen, whilst the pattern left by the vortices behind the rear wheel of the tandem-wheel case in Figure 10(b) is less clear and the size of the vortices appear to be different. Also the separation line on the bottom part of the rear wheel has a different shape from that of the single wheel case, which in this case can be associated with the slight asymmetry of the flow.

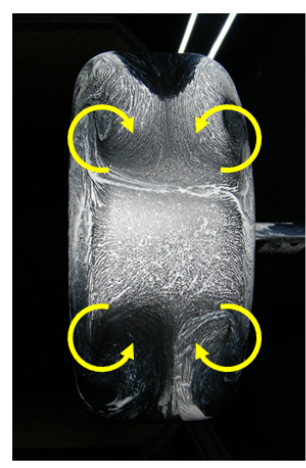

(a) Single wheel

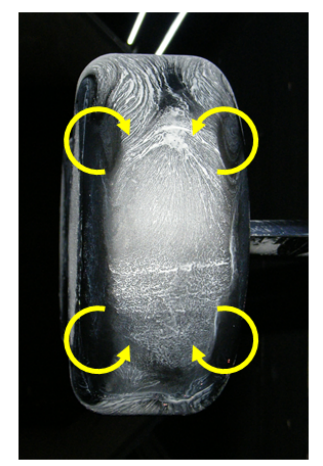

(b) Tandem wheels, $L_{w} / D_{w}=1.5, \alpha=0 \mathrm{deg}$

Figure 10: Oil flow on single wheel and tandem wheels, rear view with wake vortical system in yellow.

In Figure 11, the mean flow past tandem wheels is schematically illustrated. The schemes of the flow features were generated with additional information from CFD data (Spagnolo et al. [26]). In general, for tandem wheels, the behaviour of the vortices depends on the installation angle.

If the installation angle $\alpha$ is zero, as in Figure 11(a) the surface flow shows a system of four vortices that detach from the front wheel and impinge on the rear wheel mixing with the boundary layer on the surface. Two adjacent vortices always contain streamwise vorticity of opposite sign. At low installation angles, a system of four vortices detaching also from the rear wheel is found. However, the vortices from the front wheel interact with the boundary layer of the rear wheel, making the flow on the rear wheel substantially different from the flow of an isolated wheel. If no tripping devices are applied on the wheel surface, a laminar separation bubble is present on each side of the front wheel, and two additional laminar separation bubbles are present on the top and the bottom part of the wheel, whilst the flow on the rear wheel is fully turbulent because it 
is fully immersed in the turbulent wake generated by the front wheel.

If the rear wheel is mounted with a high installation angle, such as in Figure 11(b) where $\alpha=20 \mathrm{deg}$, the vortices detaching from the bottom of the front wheel do not impinge on the rear wheel. In this case, on the rear wheel, an additional system of four vortices is generated. In the cases where the rear wheel is not mounted at $\alpha=0 \mathrm{deg}$, the flow features on the front wheel are not symmetrical. Also, the rear wheel is partly immersed in the wake of the front wheel, thus laminar features are expected in the areas where clean flow is impinging on the rear wheel. For instance in Figure 11(b) only the top recirculation bubble is present on the rear wheel because the bottom part is partly immersed in the wake of the front wheel.

A longitudinal plane of the velocity field was acquired with PIV measurements in various configurations at constant- $z$ planes $(z=0)$. The resulting mean velocity magnitude past the single wheel is shown in Figure 12(a) and the tandem-wheel case $L_{w}=1.5 D_{w}$ at $\alpha=0 \mathrm{deg}$ is presented in Figure 12(b) The mean field wakes of the single and tandem wheels at zero installation angle have similar shapes, but the 2D turbulent kinetic energy shows higher levels of fluctuations in the tandem-wheel case. When the installation angles increases, the wake becomes larger as in the tandem wheel case $L_{w}=1.3 D_{w}$ at $\alpha=20 \mathrm{deg}$ in Figure 12(c), Two wakes can be recognised in the velocity fields. The wake with lower mean velocity and higher fluctuations levels is the wake past the rear wheel, whilst the other trace is left by the front wheel (which is positioned below in the figure), with higher mean velocity and lower 2D turbulent kinetic energy. 


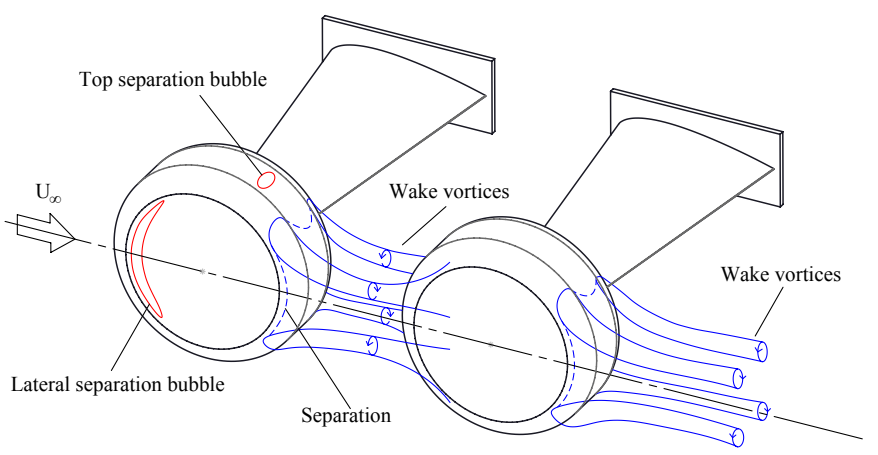

(a) $\alpha=0 \operatorname{deg}$

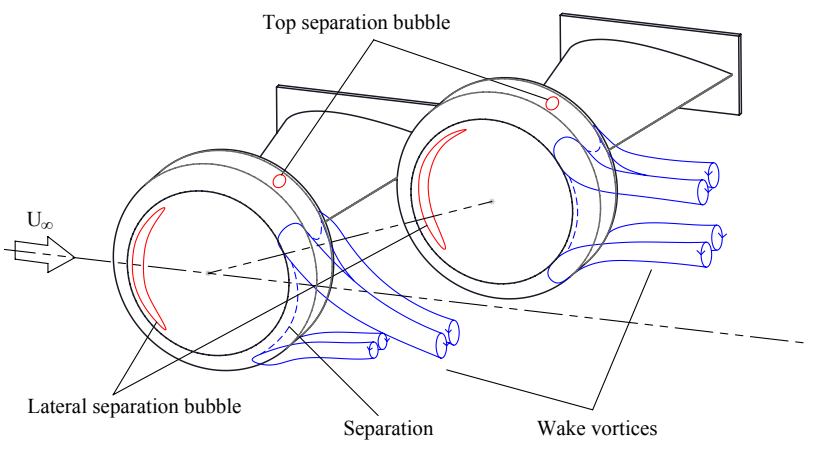

(b) $\alpha=20 \operatorname{deg}$

Figure 11: Schematics of mean flow features on tandem wheels at $L_{w} / D_{w}=1.5$ and two different installation angles. 

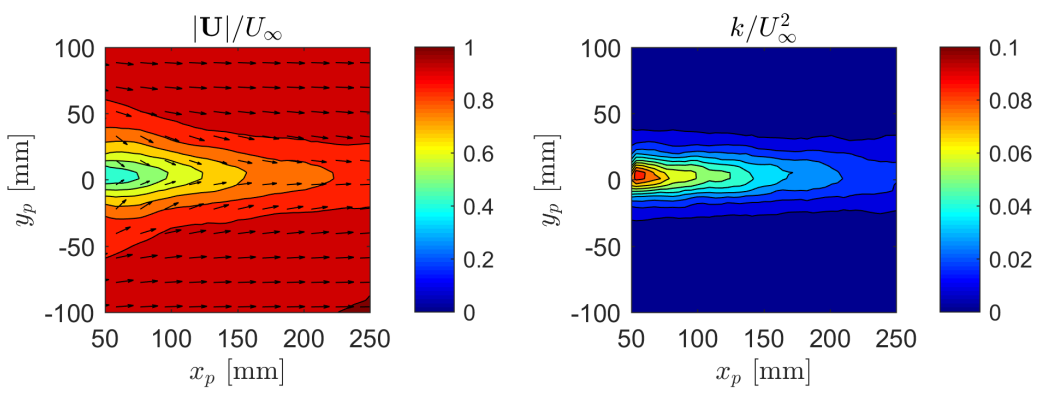

(a) Single wheel
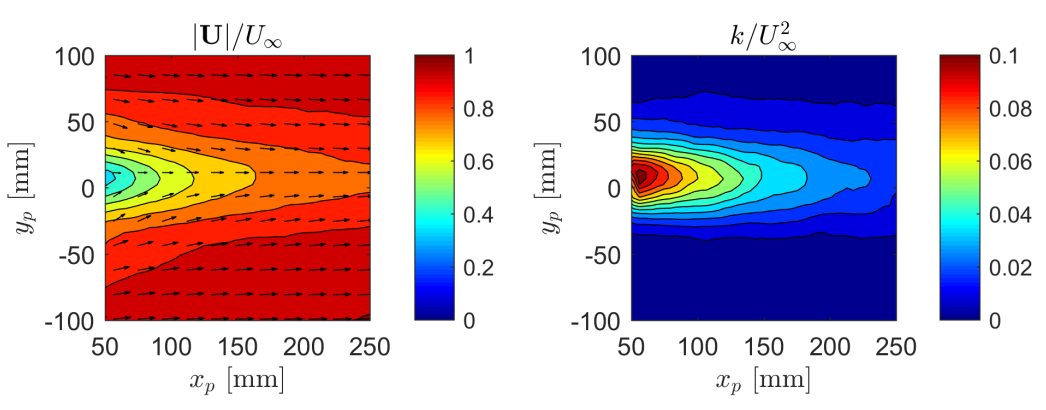

(b) Tandem wheels $L_{w}=1.5 D_{w}, \alpha=0 \mathrm{deg}$
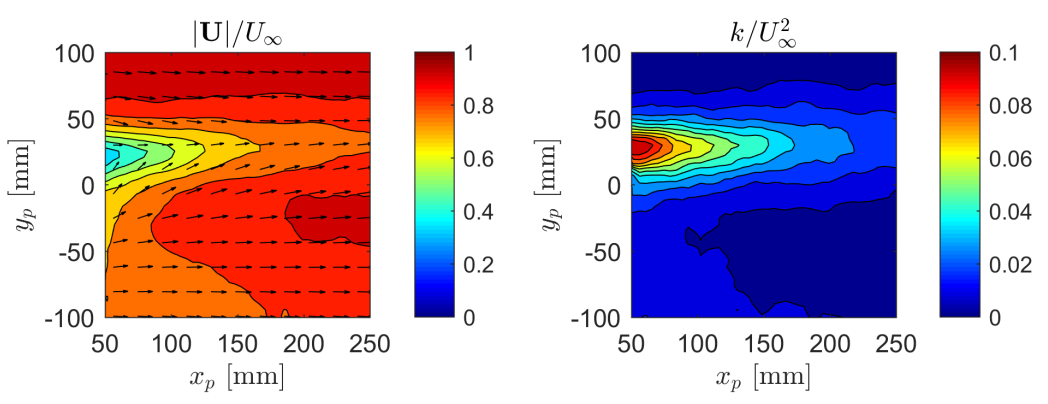

(c) Tandem wheels $L_{w}=1.3 D_{w}, \alpha=20 \mathrm{deg}$

Figure 12: Mean velocity magnitude $|\mathbf{U}|$ and 2D turbulent kinetic energy $k$ fields in nondimensional form for the $x-y$ plane along the wheel mid-plane for single wheel and tandem wheels $\left(\operatorname{Re}_{D} \approx 4.8 \times 10^{5}\right)$. 


\section{3. $P O D$ modes}

To enhance the wake flow unsteady analysis, POD modes were extracted from the PIV images using the technique detailed in Section 2.5. The first three POD modes are illustrated in Figure 13 for the single wheel and the tandem wheels at $\alpha=0$ and $L_{w}=1.5 D_{w}$.

The first mode (Figure 13(a) and Figure $13(\mathrm{~b})$ is similar in both cases, and involves a coherent vertical displacements ( $y$-direction) on a large portion of the frame. It is a flapping mode that can be identified from time series of the velocity field in the wake. It is the most energetic mode containing $25 \%$ and $42 \%$ of the total energy of the single wheel and the tandem wheels, respectively. Being the most energetic mode involving vertical displacements in the wake, the first mode can be considered responsible for generating most of the fluctuations of the lift coefficient.

The second (Figure 13(c) and third mode (Figure 13(e) of the single wheel can be identified also in the tandem wheels, but their shapes appear in an inverted order (third and second, respectively in Figure 13(f) and Figure 13(c)p. The second mode for the single wheel consists of two areas of opposite vertical velocity located in two different streamwise positions. This is the mode that mainly contributes to the sinusoidal shape of the wake that can be seen in some frames of the PIV. This mode is associated with the downstream motion of the wake structures. The third mode for the single wheel is a symmetric pulsating mode of flow that alternately converges and diverges toward the wake centreline ( $y_{p}=0$ in the pictures).

The inverted position of the modes in the tandem wheels and the higher energy content of the first mode are indicative of the lower importance of the coherent structures of Figure 13(c) or Figure 13(f) in the tandem-wheel case. In fact, the lower relative energy of the other modes is indicative of the lower importance of these structures in the tandem-wheel case with respect to the single-wheel case.

The other modes (not shown) present multiple areas with non-zero vertical velocities, representing the downstream motion of the structures observed in the second mode for single wheel and in the third mode for the tandem wheels. The wake of the tandem wheels shows a certain level of asymmetry, which is due to the asymmetry of the wake that deforms the POD modes too.

The modes energy content is given in Figure 14(a) and Figure 14(b) for the single wheel and the tandem wheels respectively. The figures show the relative importance of the modes, in decreasing order. The energy of the first mode is higher for the tandem wheels than for the single wheel because the unsteadiness of the flapping movement in the wake is more relevant in the tandem-wheel case. In Figure 15, the convergence plot of the two cases shows a faster convergence of the tandem wheels, mainly because of the higher energy content of the first mode.

In summary, the POD technique could provide a description of the most energetic mode in the wake (the flapping mode), showing that its relative importance is higher in the tandem-wheel case. 


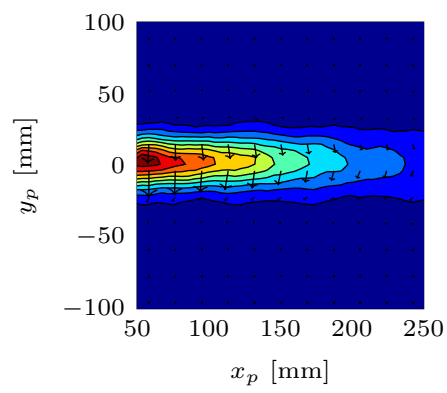

(a) Single-wheel POD Mode 1, $25.4 \%$ energy

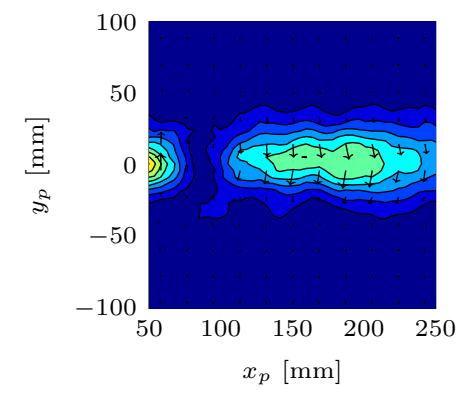

(c) Single-wheel POD Mode 2, $8.2 \%$ energy

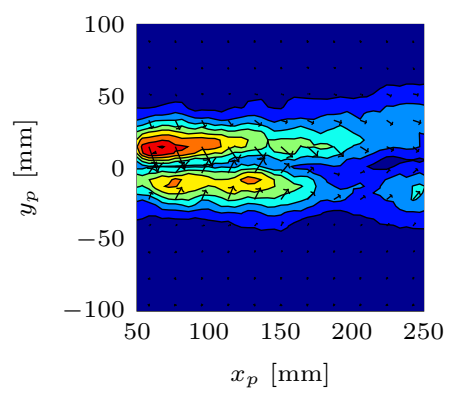

(e) Single-wheel POD Mode 3, $4.8 \%$ energy

Figure 13: Comparison of the first three POD modes for single (left) and tandem wheels (right), contours of dimensionless velocity magnitude.

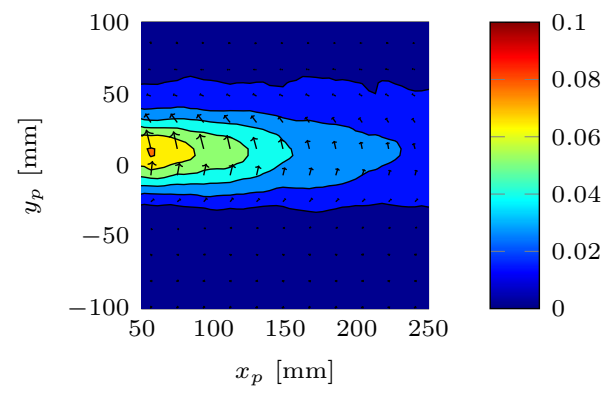

(b) Tandem-wheel POD Mode 1, 41.6\% energy

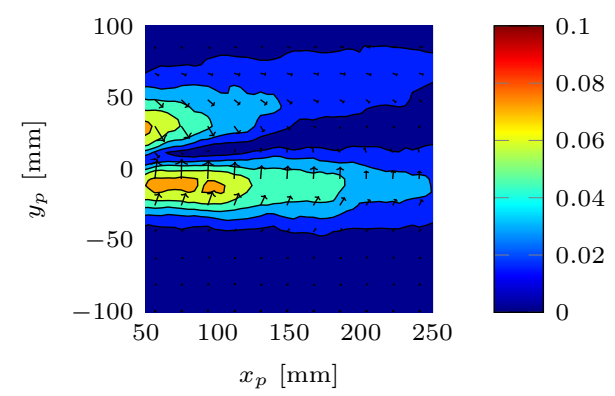

(d) Tandem-wheel POD Mode 2, $8.2 \%$ energy

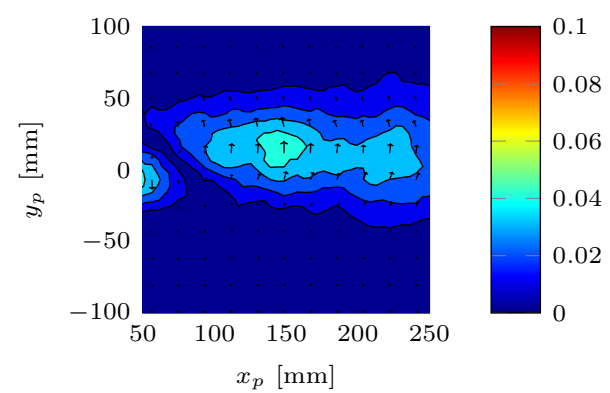

(f) Tandem-wheel POD Mode 3, 4.9\% energy 


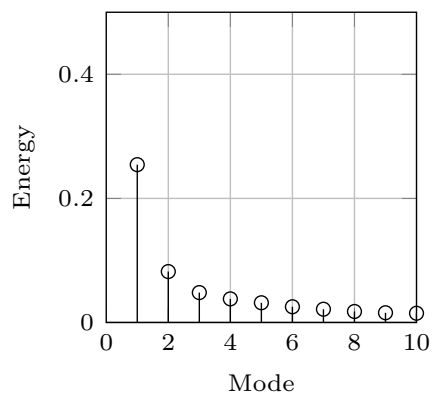

(a) Single wheel

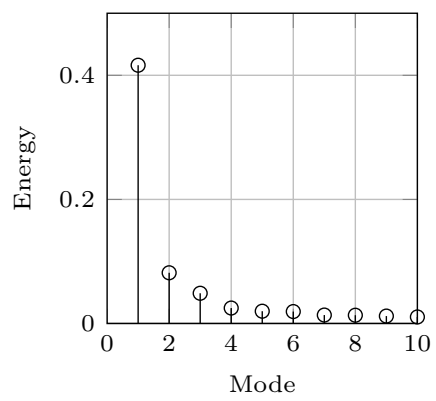

(b) Tandem wheels

Figure 14: POD energy contents

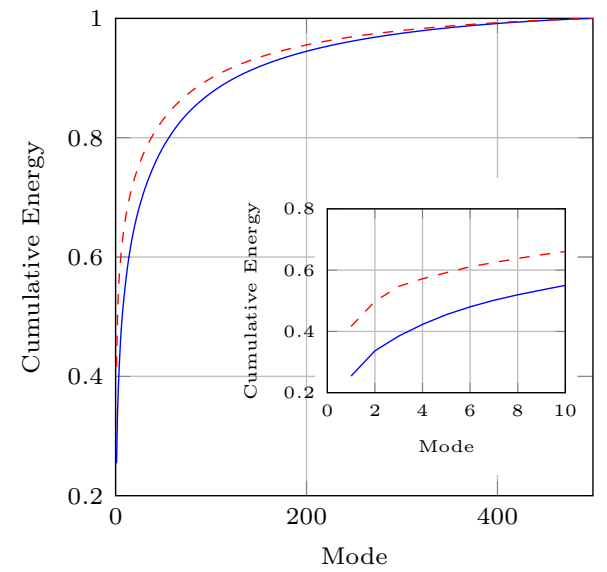

Figure 15: POD cumulative energy contents for single wheel $\square$ and tandem wheels --- . 


\subsection{Effects of inter-axis distance and installation angle on tandem wheels}

To understand the dependency on the configurations of the mean forces and their unsteady fluctuations, tests were repeated in a range of $L_{w} / D_{w}$ ratio and installation angle $\alpha$. The front-wheel drag coefficient $\bar{C}_{D}^{f}$ and rear-wheel drag coefficient $\bar{C}_{D}^{r}$ are summarized in Table 1 , together with the total drag coefficient $\bar{C}_{D}^{t}$ from both wheels at $R e_{D}=4.8 \times 10^{5}$. The same data are also shown in Figure 16.

For the tandem-wheel case with zero installation angle, the front wheel has a lower mean drag coefficient than the rear wheel. The mean total drag of the tandem-wheel configuration is $9 \%$ lower than twice the isolated single wheel, but the rear wheel alone shows an increase of drag with respect to an isolated wheel, while the front wheel has a lower mean drag coefficient (see also Figure 3). The reason for the lower mean drag on the front wheel is the high pressure region on the fore part of the rear wheel, which also affects the pressure on the downstream part of the front wheel, leading to a lower mean drag coefficient. The high mean drag coefficient of the rear wheel is explained by the higher intensity of the four vortices detaching from the rear wheel compared to the single wheel case. The vortices are stronger because they are the combination of the vortices formed on the front wheel and the vortices formed on the rear wheel. The vortices form suction areas on the wheel shoulders that contribute to the increase of drag.

Table 1: Effect of the geometric parameters on mean loads $\left(R e_{D}=4.8 \times 10^{5}\right)$.

\begin{tabular}{|c|c|c|c|c|c|c|c|}
\hline \multicolumn{3}{|c|}{ Configuration } & \multirow{2}{*}{$\bar{C}_{D}^{f}$} & \multirow{2}{*}{$\bar{C}_{L}^{f}$} & \multirow{2}{*}{$\bar{C}_{D}^{r}$} & \multirow{2}{*}{$\bar{C}_{L}^{r}$} & \multirow{2}{*}{$\bar{C}_{D}^{t}$} \\
\hline Name & $\alpha[\mathrm{deg}]$ & $L_{w} / D_{w}$ & & & & & \\
\hline Single wheel & & & 0.229 & 0.004 & & & 0.229 \\
\hline \multirow[t]{6}{*}{ Tandem wheels } & 0 & 1.1 & 0.093 & -0.137 & 0.305 & -0.004 & 0.398 \\
\hline & 0 & 1.3 & 0.060 & 0.048 & 0.337 & -0.003 & 0.397 \\
\hline & 0 & 1.5 & 0.082 & -0.002 & 0.336 & 0.024 & 0.418 \\
\hline & 10 & 1.5 & 0.124 & 0.022 & 0.313 & 0.035 & 0.437 \\
\hline & 20 & 1.3 & 0.181 & -0.005 & 0.384 & 0.032 & 0.565 \\
\hline & 20 & 1.5 & 0.190 & 0.010 & 0.320 & 0.021 & 0.510 \\
\hline
\end{tabular}

A change in the inter-axis distance has the main effect of redistributing the drag coefficient between the two wheels, without dramatically affecting the total drag coefficient (differences within 5\%). The flow is asymmetric for small inter-axis distances, which is revealed by the non-zero mean lift coefficients.

At $\alpha=0 \mathrm{deg}$, within the tested range, $\bar{C}_{L}^{f}$ decreases in magnitude with increasing $L_{w} / D_{w}$ ratio, whilst the rear lift coefficient magnitude increases. The front-wheel mean lift coefficient $\bar{C}_{L}^{f}$ is not zero in the lowest and in the medium distances $\left(L_{w} / D_{w}=1.1\right.$ and 1.3) in spite of a zero installation angle. Similar asymmetric flows were previously observed by Khorrami et al. [10] on tandem cylinders, in similar Reynolds number ranges. On the contrary, with the highest $L_{w} / D_{w}=1.5$ the flow is symmetric, i.e. the mean lift is small for both wheels. The mean rear lift coefficient is never as high as the front lift coefficient 

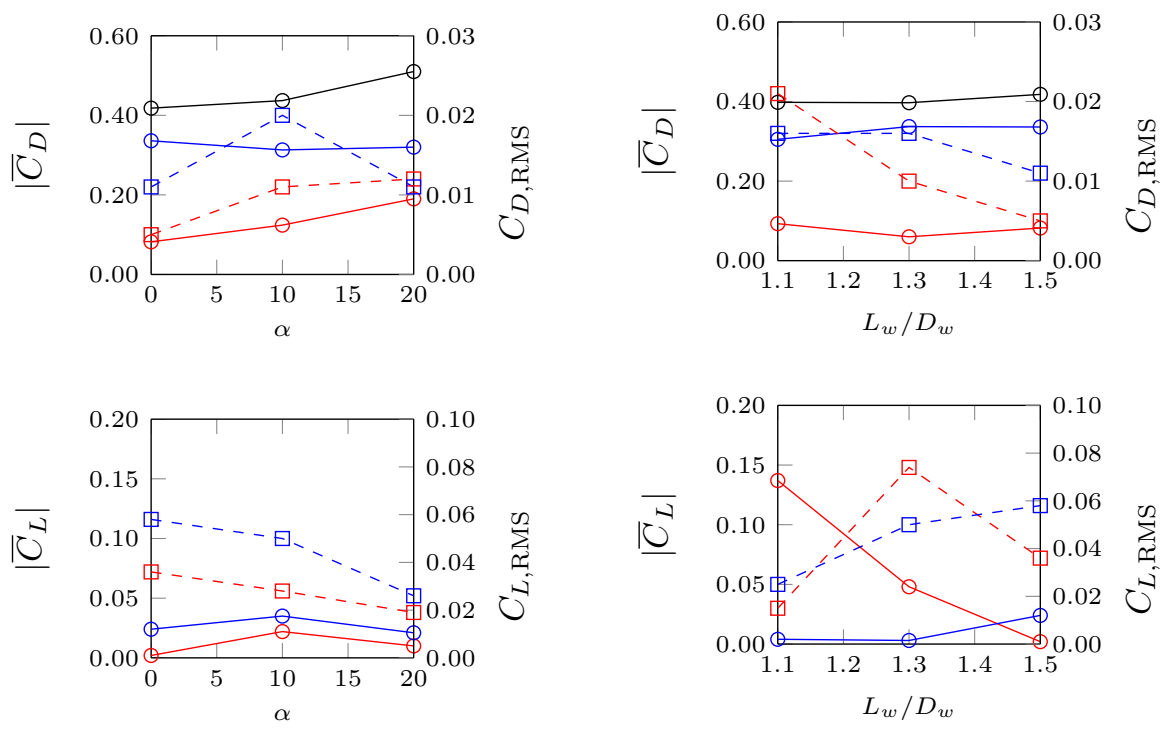

Figure 16: Mean and RMS force coefficients $\bar{C}_{D}$ and $\left|\bar{C}_{L}\right|$ as a function of the geometric parameters $L_{w} / D_{w}$ and $\alpha$ ): front wheel $\square$, rear wheel $\square$, and total $\square$. Solid lines are mean values, dashed lines are RMS values $\left(R e_{D}=4.8 \times 10^{5}\right)$. Left-hand side graphs are with constant $L_{w} / D_{w}=1.5$ and right-hand side graphs are with constant $\alpha=0$ deg.

in the inter-axis distance of $L_{w}=1.1 D_{w}$. Increasing the installation angle from $0 \mathrm{deg}$ to $20 \mathrm{deg}$, the total drag coefficient increases by $22 \%$ with $L_{w}=1.5 D_{w}$ and by $42 \%$ with $L_{w}=1.3 D_{w}$. In fact, a global drag increase is expected due to the larger projected frontal area. The increase is mainly on the front wheel. As the installation angle increases, the front wheel loses the high back pressure due to the impingement of its wake on the rear wheel. Instead stronger vortices generate low pressure region in the wake. Change in the drag coefficient is more evident for $L_{w} / D_{w}=1.3$.

For the tandem-wheel case, the RMS values of aerodynamic loads are given in Table 2. The standard deviations are generally higher for the tandem-wheel case than the single-wheel case. The lift coefficient RMS is generally higher than the respective drag coefficient RMS. The configurations that provide the lowest levels of RMS overall are the cases with high installation angle, in spite of the higher mean drag coefficient. When comparing configurations at $\alpha=0 \mathrm{deg}$, a higher inter-axis distance generally corresponds to a lower RMS, especially on the front wheel.

A peak was observed in the PSD of the lift coefficient (not shown here), in the range that was used for the calculation of the filtered RMS. The peak is not considered structural, since it did not result from the vibration test and it changes frequency with the velocities. The peak occurs at a Strouhal number $S t \approx 0.07$ in the range $3.6 \times 10^{5}<R e_{D}<5.7 \times 10^{5}$. A smaller peak at $S t \approx$ 
Table 2: Effect of the geometric parameters on loads standard deviations $\left(R e_{D}=4.8 \times 10^{5}\right)$.

\begin{tabular}{|c|c|c|c|c|c|c|}
\hline \multicolumn{3}{|c|}{ Configuration } & \multirow{2}{*}{$C_{D, \mathrm{RMS}}^{f}$} & \multirow{2}{*}{$C_{L, \mathrm{RMS}}^{f}$} & \multirow{2}{*}{$C_{D, \mathrm{RMS}}^{r}$} & \multirow{2}{*}{$C_{L, \mathrm{RMS}}^{r}$} \\
\hline Name & $\alpha[\mathrm{deg}]$ & $L_{w} / D_{w}$ & & & & \\
\hline Single wheel & & & 0.005 & 0.032 & & \\
\hline \multirow[t]{6}{*}{ Tandem wheels } & 0 & 1.1 & 0.021 & 0.015 & 0.016 & 0.025 \\
\hline & 0 & 1.3 & 0.010 & 0.074 & 0.016 & 0.050 \\
\hline & 0 & 1.5 & 0.005 & 0.036 & 0.011 & 0.058 \\
\hline & 10 & 1.5 & 0.011 & 0.028 & 0.020 & 0.050 \\
\hline & 20 & 1.3 & 0.013 & 0.017 & 0.012 & 0.031 \\
\hline & 20 & 1.5 & 0.012 & 0.019 & 0.011 & 0.026 \\
\hline
\end{tabular}

0.07 and a secondary peak at a Strouhal number $S t \approx 0.14$ can be observed in the drag coefficient too. The phenomenon is expected to be related with the transitional flow, because the tripped configurations do not show the same feature. 27.

In Figure 17, the pressure coefficients $C_{p}$ of the three tandem-wheel configurations are compared to analyse the effect of the inter-axis distance and the installation angle. The two cases with $\alpha=0 \mathrm{deg}$ show differences in mean values only in the fore part of the rear wheel. The case with the shorter inter-axis distance $\left(L_{w} / D_{w}=1.3\right)$ has a lower pressure at $\theta=0 \mathrm{deg}$ because the flow velocity between the two wheels remains low, generating lower dynamic pressure. Except for the region in the proximity of $\theta=0 \mathrm{deg}$, the two considered cases at $\alpha=0 \mathrm{deg}\left(L_{w} / D_{w}=1.3\right.$ and $\left.L_{w} / D_{w}=1.5\right)$ are similar, showing symmetric high-pressure areas $\left(C_{p} \approx 0.8\right)$ at $30 \mathrm{deg}$ and $330 \mathrm{deg}$ on the rear wheel, where the flow separated from the front wheel impinges on the rear wheel. The short distance increases the unsteady interactions between the front and the rear wheels, as it can be seen from the RMS pressure coefficients. Most of the difference is concentrated in the areas between the two wheels, not only on the rear wheel on the impingement areas of the wake from the front wheel, but also on the aft part of the front wheel. The presence of the rear wheel affects the unsteady flow on the front wheel.

The pressure coefficients $C_{p}$ for the same inter-axis distance $L_{w} / D_{w}=1.5$ and two angles of attack ( $\alpha=0 \mathrm{deg}$ and $\alpha=20 \mathrm{deg}$ ) are also compared in Figure 17. The mean flow past the rear wheel at $\alpha=0 \mathrm{deg}$ is substantially different from the flow past the rear wheel at $\alpha=20 \mathrm{deg}$. At such a high installation angle, the flow past the rear wheel shows a trend similar to the flow past a single wheel: only one stagnation point in the front and a recirculation bubble at approximately $\theta=120 \mathrm{deg}$. The flow on the wheels is clearly not symmetrical due to the wake past the front wheel. The maximum suction peak at $\theta=270 \mathrm{deg}$ has a lower $\bar{C}_{p}$ than the suction peak $\theta=90 \mathrm{deg}$. Most of the unsteady content is concentrated around $\theta=180 \mathrm{deg}$, similarly to the trend observed on the single wheel, but with slightly higher values. Overall, in the case with $\alpha=20 \mathrm{deg}$ the curve of the RMS on the rear wheel shows lower RMS than that in the configuration at $\alpha=0 \mathrm{deg}$. 
In Figure 17 a comparison with the single wheel case is also provided. The front wheel in tandem configuration shows a mean drag decrease with respect to the single wheel because of the higher pressure in the rear part of the front wheel. The rear wheel shows both areas with increased and decreased pressure with respect to the single wheel, therefore the centreline pressure profiles cannot be used to explain the slight difference in mean drag coefficient.
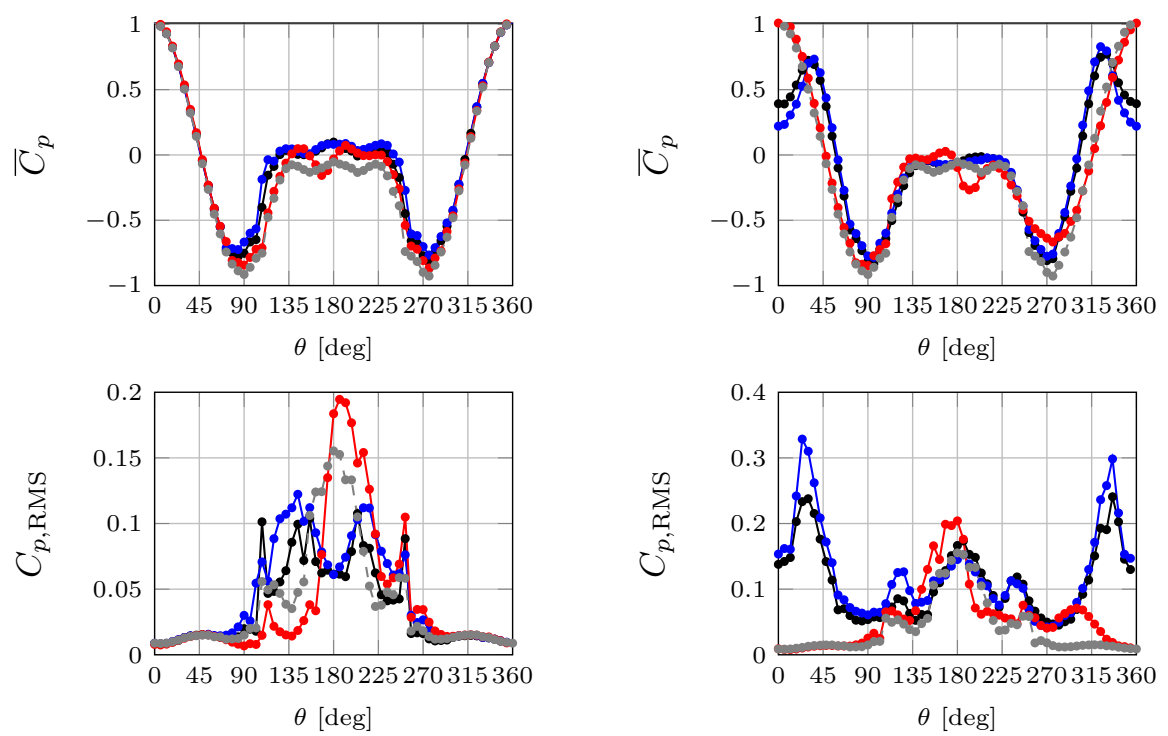

(a) Front wheel

(b) Rear wheel

Figure 17: Mean and RMS values of the pressure coefficients $C_{p}$ along the rear wheel center line, comparing the tandem wheels configurations $L_{w} / D_{w}=1.3 \square, L_{w} / D_{w}=1.5 \square$, both at $\alpha=0 \mathrm{deg}$, and $L_{w} / D_{w}=1.5$ at $\alpha=20 \mathrm{deg} \longrightarrow$. Untripped single wheel $\rightarrow-\infty$ is also shown for comparison.

\section{Conclusions}

Aerodynamic loads, surface pressures and velocity fields in the wake were experimentally measured for the flow past single and tandem wheels. The tandemwheel model represents half of a simplified four-wheel landing gear. The aerodynamic force measurements were validated with an in-situ vibration test on the model structure, to estimate the dynamic response of the balance. The wind-tunnel tests were performed in the $2.1 \mathrm{~m} \times 1.5 \mathrm{~m}$ wind tunnel at the University of Southampton, for different inter-axis distances and installation angles. Overall, the tested model shows evidences of a low sensitivity to Reynolds number in this transitional regime, which is characterised by the presence of both laminar and turbulent flow features. One of the main findings of this study is the limited effect of the inter-axis distance on the global mean drag coefficient 
in the tested range of $L_{w} / D_{w}$ (inter-axis distance over diameter ratio) between 1.1 and 1.5, while the effect on the standard deviations is more evident. The general trend is toward higher mean drag coefficient and a lower RMS of force coefficients with increasing inter-axis distance. In spite of zero installation angle, a non-symmetric mean flow was found for small $L_{w} / D_{w}$ ratios. The effect of the installation angle is relevant both for mean and RMS values. Higher installation angles lead to higher mean drag coefficients but to lower RMS of the force coefficients. PIV and oil-flow images allowed the identification of the four vortices in the wake, along with the identification of the POD modes that are responsible for the generation of the force fluctuations. A transverse plane of the flow field (not measured here) would allow the identification of the exact position of the vortices.

\section{Acknowledgments}

This work is funded by the UK Aerospace Technology Institute under the Advanced Landing Gear Aero-Loads and Aero-Noise Prediction (ALGAAP) project. In particular, the authors are thankful to Alan Shepherd (Airbus) for his advice and support. The wind-tunnel staff at the University of Southampton are kindly acknowledged for their technical support.

\section{References}

[1] Baban, F., So, R., Ötügen, M., 1989. Unsteady forces on circular cylinders in a cross-flow. Experiments in Fluids 7, 293-302.

[2] Berkooz, G., Holmes, P., Lumley, J. L., 1993. The proper orthogonal decomposition in the analysis of turbulent flows. Ann. Rev. Fluid Mech. 25, $539-575$.

[3] Bernero, S., Fiedler, H. E., 2000. Application of particle image velocimetry and proper orthogonal decomposition to the study of a jet in a counterflow. Experiments in Fluids, S274-S281.

[4] Capece, V., Fleeter, S., 1986. The unsteady aerodynamics of a first stage stator vane row. Experiments in Fluids 4, 72-78.

[5] De Lucca, N., Gordeyev, S., Jumper, E., Thordahl, J., Wittich, D. J., 2013. The estimation of the unsteady aerodynamic force applied to a turret in flight AIAA Paper 2013-3136.

[6] Dobrzynski, W., March-April 2010. Almost 40 years of airframe noise research: what did we achieve? Journal of Aircraft 47 (2), 353-367.

[7] Grant, I., Owens, E. H., April 1990. Confidence interval estimates in PIV measurements of turbulent flows. Applied optics 29 (10), 1400-1402. 
[8] Hu, H., Yang, Z., Sarkar, P., 2012. Dynamic wind loads and wake characteristics of a wind turbine model in an atmospheric boundary layer wind. Experiments in Fluids 52, 1277-1294.

[9] Humphreys, W. M. J., Brooks, T. F., 2007. Noise spectra and directivity for a scale-model landing gear AIAA Paper 2007-3458.

[10] Khorrami, M. R., Choudhari, M. M., Lockard, D., Jenkins, L. N., McGinley, C. B., 2007. Unsteady flowfield around tandem cylinders as prototype component interaction in airframe noise. AIAA Journal 45 (8), 1930-1941.

[11] Kurtulus, D., Scarano, F., David, L., 2007. Unsteady aerodynamic forces estimation on square cylinder by TR-PIV. Experiments in Fluids 42, 185196.

[12] Lazos, B. S., 2002. Mean flow features around the inline wheels of fourwheel landing gear. AIAA Journal 40 (2), 193-168.

[13] Lockard, D. P., 2011. Summary of the tandem cylinder solutions from the benchmark problems for airframe noise computations - I workshop AIAA Paper 2011-353.

[14] Lu, F., 2010. Surface oil flow visualization. Eur. Phys. J. Special Topics 182, 51-63.

[15] Manoha, E., Bulté, J., Caruelle, B., 2008. Lagoon: An experimental database for the validation of CFD/CAA methods for landing gear noise prediction AIAA Paper 2008-2816.

[16] McManus, J., Zhang, X., 2005. A computational study of the flow around an isolated wheel in contact with the ground. Journal of Fluids Engineering $128(3)$.

[17] Meyer, K. E., Cavar, D., Pedersen, J. M., September 2007. Pod as tool for comparison of piv and les data 7th International Symposium on Particle Image Velocimetry, Rome, Italy.

[18] Nitsche, W., Mirow, P., 1989. Piezo-electric foils as a means of sensing unsteady surface forces. Experiments in Fluids 7, 111-118.

[19] Okajima, A., 1979. Flows around two tandem circular cylinders at very high Reynolds numbers. Bulletin of the Japan Society of Mechanical Engineers 166 (7), 504-511.

[20] Piersol, A. G., Paez, T. L., 2010. Harris' Shock and vibration handbook, 6th Edition. McGraw-Hill Handbooks.

[21] Robinson, M., Hannemann, K., 2006. Short duration force measurements in impulse facilities AIAA Paper 2006-3439. 
[22] Sawanda, H., Suda, S., 2011. Study on aerodynamic force acting on a sphere with and without boundary layer trips around the critical Reynolds number with a magnetic suspension balance system. Experiments in Fluids 50, 271284.

[23] Schuster, D. M., Byrd, J. E., 2003. Transonic unsteady aerodynamics of the F/A-18E at conditions promoting abrupt wing stall AIAA Paper 20030593.

[24] Shur, M. L., Spalart, P. R., Strelets, M. K., Travin, A., 2008. A hybrid RANS-LES model with delayed DES and wall-modeled LES capabilities. International Journal of Heat and Fluid Flow 29, 1638-1649.

[25] Sirovich, L., 1987. Turbulence and the dynamics of coherent structures. Part I: coherent structures. Quaterly of applied mathematics 45 (3), 561571.

[26] Spagnolo, S., Zhang, X., Hu, Z., Angland, D., 2015. Numerical simulations of single and tandem wheels for aerodynamic loads prediction AIAA Paper 2015-3066.

[27] Spagnolo, S., Zhang, X., Hu, Z., Stalnov, O., Angland, D., 2015. Unsteady force and flow features of single and tandem wheels AIAA Paper 2015-0261.

[28] Stalnov, O., Windiate, S., Angland, D., Zhang, X., Ashworth, R., 2013. On the contribution of individual components to landing gear loads and noise AIAA Paper 2013-3153.

[29] Sumner, D., 2010. Two circular cylinders in cross-flow: A review. Journal of Fluid and Structures 26, 849-899.

[30] Tirunagari, S., Vuorinen, V., Kaario, O., Larmi, M., 2012. Analysis of proper orthogonal decomposition and dynamic mode decomposition on LES of subsonic jets. CSI Journal of Computing 1 (3), 2:19-2:24.

[31] Tropea, C., Yarin, A. L., Foss, J. F. (Eds.), 2003. Springer Handbook of Experimental Fluid Mechanics. Springer.

[32] Venkatakrishnan, L., Karthikeyan, N., Mejia, K., November 2012. Experimental studies on a rudimentary four-wheel landing gear. AIAA Journal 50 (11), 2435-2447.

[33] Xiao, Z., Luo, K., May 2013. Numerical simulations of tandem cylinders with subcritical spacing AIAA Paper 2013-2209.

[34] Zawodny, N. S., Liu, F., Yardibi, T., Cattafesta, L., Khorrami, M. R., Neuhart, D. H., Van de Ven, T., 2009. A comparative study of a 1/4-scale Gulfstream G550 aircraft nose gear model AIAA Paper 2009-3153.

[35] Zdravkovich, M. M., Flaherty, A. J., Pahle, M. G., Skelhorne, I. A., 1998. Some aerodynamic aspects of coin-like cylinders. J. Fluid Mech. 360, 73-84. 
[36] Zhang, X., Ma, Z., Smith, M., Sanderson, M., Bissessur, P., 2013. Aerodynamic and acoustic measurements of a single landing gear wheel AIAA Paper 2013-2160. 